\title{
Method of Constructing an Asymmetric Human Bronchial Tree in Normal and Pathological Cases
}

\author{
Medvedev A.E.*
Khristianovich Institute of Theoretical and Applied Mechanics SB RAS, Novosibirsk, Russia Novosibirsk State University, Novosibirsk, Russia

\begin{abstract}
The goal of the study is the analytical design of the full asymmetric human bronchial tree (irregular dichotomy) for healthy patients and patients with obstructive pulmonary diseases. For this purpose, the author has derived the special analytical formulas. All surfaces of the bronchial tree are matched with the secondorder smoothness (there are no acute angles or ribs). The geometric characteristics of the human bronchial tree in the pathological case are modeled by a "starry" shape of the inner structure of the bronchus; a level of the pathology is defined by two parameters: bronchus constriction level and level of distortion of the cylindrical shape of the bronchus. Closed analytical formulas allow a researcher to construct the human bronchial tree of an arbitrary complexity (up to alveoli); moreover, the parametric dependences make it possible to specify any desirable level of airway obstruction.
\end{abstract}

Key words: bronchial tree, simulation, human lungs, respiratory system, bifurcation, lung disease.

\section{INTRODUCTION}

The human respiratory system consists of the upper (nose, nasal pharynx, and larynx) and lower (trachea, bronchial tree, and alveoli) air passages. The tree-like structure of the human bronchial tree has 24 branching points (bifurcations). There are approximately $2^{23}=8,388,608$ alveoli at the end of the bronchial tree branches.

To model respiratory processes in human lungs, it is necessary first of all to design a threedimensional model of the bronchial tree. As was noted in [1], the regular dichotomy model is suitable for many cases of the general behavior of the human respiratory system. Regular dichotomy is a symmetric model of the bronchial tree where all elements (branches) in one generation of bifurcations have identical sizes and bronchus deflection angles. Such a method of symmetric bronchial tree design was proposed in [2].

To describe real (or close to real) human lungs, it is necessary to use irregular dichotomy [1], where the symmetry of bronchus branches in one generation of bifurcations is violated.

The upper part of the bronchial tree with well-described morphometric data is reviewed in several papers [3-15]. An advanced review of these investigations can be found in [2]. It should be only noted here that it is difficult to replicate the bronchial tree design of these researchers because: (1) not all morphometric data are provided in publications, (2) the algorithm of bifurcation design in the 3D simulation code used by researchers is not described in sufficient detail, and (3) the algorithm of construction of an individual bifurcation is too complicated (a detailed description of construction of an individual bifurcation in [5] takes eight pages).

The listed complications claim for a method based on simple analytical formulas to design an individual bifurcation and assemble several bifurcations into a tree. This method does not

*medvedev@itam.nsc.ru 
require manual operations to design a bronchial tree and can be readily reproduced by other researchers. An algorithmic method of constructing regular dichotomy on the basis of simple analytical formulas was proposed in [2]. This method is extended below to the case of irregular dichotomy for an asymmetric bronchial tree. This method allows a researcher to construct a bronchial tree of any complexity. The bottleneck of this method is the lack of detailed morphometric data for small bronchi and constraints in the possibility of machine presentation of a three-dimensional model of such a tremendous size.

\section{BRONCHIAL TREE DESIGN AND CONSTRUCTION}

To design the full human bronchial tree, the author constructed an individual asymmetric bifurcation. The full set of the bronchi can be obtained by changing of the number of this bifurcation. The bronchus position in the bronchial tree is also defined analytically using the coordinate transformation in accordance with the bronchus position in the tree.

\section{Change in the bronchus shape}

The internal cross section of the bronchi is not rigorously circular even in the nonpathological case $[16,17]$. A brief review of the histological structure of the bronchi can be found in [2]. It should be only noted that the internal cross section of the bronchi has a "starry" shape.

The function that describes the cross-sectional shape of the bronchi of healthy patients and patients with obstructive pulmonary diseases can be written in the following form [2]:

$$
f(\varphi)=\left[C-s_{\mathrm{ob}} \sin ^{2}\left(\frac{n_{\mathrm{ed}}}{2} \varphi\right)\right] \sqrt{A_{\text {bronch }}},
$$

where $A_{\text {bronch }}$ is the degree of decreasing of the cross-sectional area of the obstructed bronchus as compared to that of the healthy bronchus $\left(0<A_{\mathrm{bronch}} \leq 1\right), s_{\mathrm{ob}}$ is the normalized height of the rays of the "star" of the internal shape of the bronchus $\left(0 \leq s_{\mathrm{ob}} \leq 1\right)$, and $n_{\mathrm{ed}}$ is the number of rays in the "star" representing a bronchus internal shape. The parameter $C$ is chosen from the condition that the cross-sectional area of the bronchus remains constant as the values of $s_{\mathrm{ob}}$ and $n_{\text {ed }}$ change:

$$
\begin{aligned}
& C=\frac{s_{\mathrm{ob}}\left[2 \pi n_{\mathrm{ed}}-\sin \left(2 \pi n_{\mathrm{ed}}\right)\right]+\sqrt{\Delta}}{4 \pi n_{\mathrm{ed}}} \\
& \left(\Delta=2 \pi n_{\mathrm{ed}} s_{\mathrm{ob}}^{2} \sin \left(2 \pi n_{\mathrm{ed}}\right) \sin ^{2}\left(\pi n_{\mathrm{ed}}\right)+2 \pi^{2} n_{\mathrm{ed}}^{2}\left(8-s_{\mathrm{ob}}^{2}\right)-s_{\mathrm{ob}}^{2}\left(1-\pi n_{\mathrm{ed}}\right) \sin \left(2 \pi n_{\mathrm{ed}}\right)\right) . \text { At } s_{\mathrm{ob}}=0, \\
& f(\varphi)=1 .
\end{aligned}
$$

The equations of the cross section of the bronchus with obstructive constriction (1) are

$$
\begin{aligned}
& X^{\mathrm{B}}(\varphi)=R^{\mathrm{B}} \cos (\varphi) \cdot f(\varphi), \\
& Y^{\mathrm{B}}(\varphi)=R^{\mathrm{B}} \sin (\varphi) \cdot f(\varphi),
\end{aligned}
$$

where $\varphi \in[0,2 \pi]$, and $R^{\mathrm{B}}$ is the bronchus radius.

\section{Construction of an asymmetric bifurcation}

The equation for a torus with the distance between the circumference center and the rotation axis $R$, and the circumference radius $r$ is defined parametrically by the formulas 


$$
\begin{aligned}
& X(\varphi, \chi, R, r)=[R+r \cdot f(\varphi) \cos \varphi] \cos \chi, \\
& Y(\varphi, r)=r \cdot f(\varphi) \sin \varphi, \\
& Z(\varphi, \chi, R, r)=[R+r \cdot f(\varphi) \cos \varphi] \sin \chi,
\end{aligned}
$$

where the angles are $\varphi \in[0,2 \pi]$ and $\chi \in[-\pi, \pi]$, and $\mathrm{f}(\varphi)$ is the function of the bronchus cross section shape (1).

To construct torus (4) with a variable circumference radius $R$, we need the equation for the curve connecting two points $\left(\chi_{0}, R_{0}\right)$ and $\left(\chi_{1}, R_{1}\right)$ :

$$
\begin{aligned}
R^{M}\left(\beta, \chi ; \chi_{0}, \chi_{1}, R_{0}, R_{1}\right)= & \beta\left[\frac{R_{0}-R_{1}}{2} \sin \left(\pi\left(\frac{\chi-\chi_{0}}{\chi_{1}-\chi_{0}}+\frac{1}{2}\right)\right)+\frac{R_{0}+R_{1}}{2}\right]+ \\
& +(1-\beta)\left[\frac{\left(R_{0}-R_{1}\right) \chi+\left(R_{0} \chi_{1}-R_{1} \chi_{0}\right)}{\chi_{1}-\chi_{0}}\right],
\end{aligned}
$$

where the parameter $\beta(0 \leq \beta \leq 1)$ controls the slope of curve (5) at the end points $\left(\chi_{0}, R_{0}\right)$ and $\left(\chi_{1}, R_{1}\right)$. For $\chi=\chi_{0}$ and $\chi=\chi_{1}$, the slope of curve (5) is $(1-\beta) \frac{R_{1}-R_{0}}{\chi_{1}-\chi_{0}}$.

The design of an individual bifurcation in the projection onto the plane $y=0$ is schematically shown in Figure 1.

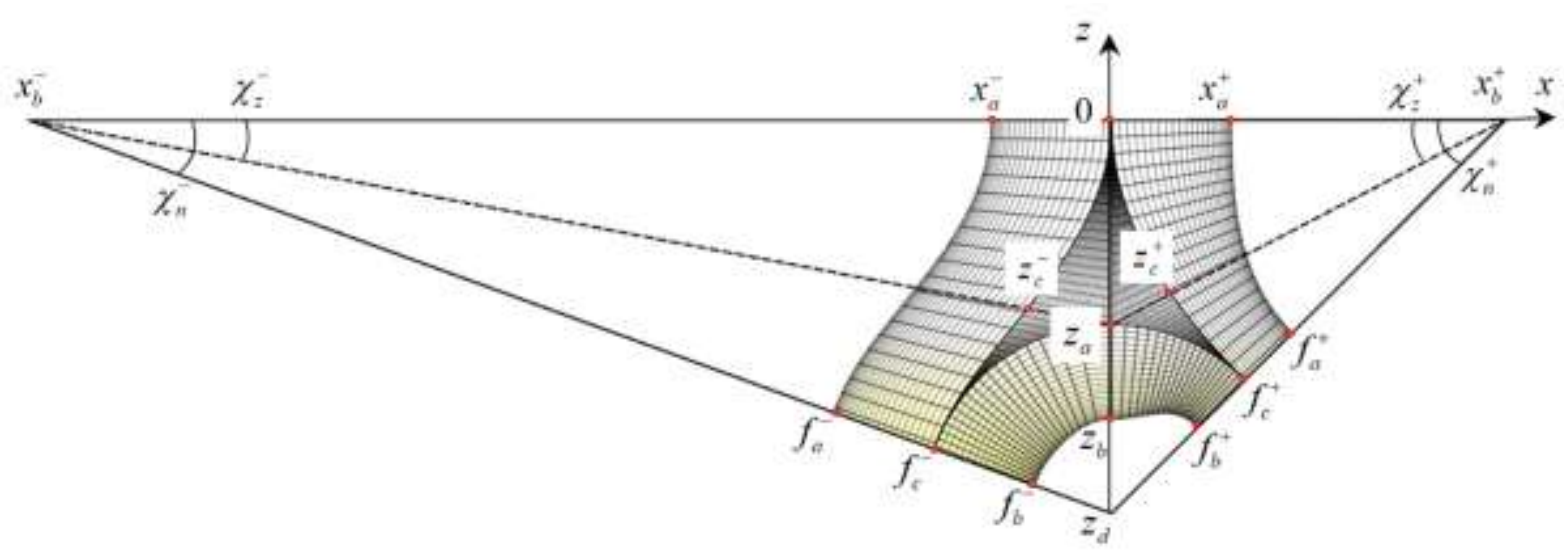

Fig. 1. Construction of the $n$-th bifurcation of the bronchial tree (the figure illustrates the construction of a circular bifurcation, and the output bronchi are not shown). General view of the three-dimensional bifurcation in the projection onto the plane $y=0$. Here $\left(0, x_{a}^{ \pm}, f_{a}^{ \pm}, f_{c}^{ \pm}\right)$is the upper torus, $\left(z_{a}, f_{c}^{ \pm}, f_{b}^{ \pm}, z_{b}\right)$ is the lower torus, $\left(0, z_{c}^{ \pm}, z_{a}\right)$ is the upper surface, and $\left(z_{a}, z_{c}^{ \pm}, f_{c}^{ \pm}\right)$is the lower surface.

The upper torus $\left(0, x_{a}^{ \pm}, f_{a}^{ \pm}, f_{c}^{ \pm}\right)$has the input radius of the generatrix (radius $r$ in formula (4)) $\left(0, x_{a}^{ \pm}\right)=R_{\text {in }}$ and the output radius $\left(f_{c}^{ \pm}, f_{a}^{ \pm}\right)=\left(f_{c}^{ \pm}, f_{b}^{ \pm}\right)=R_{\text {out }}^{ \pm}$, the torus is bounded by the angle $\chi_{n}^{ \pm}$. The distance along the arc $\left(0, z_{c}^{ \pm}, f_{c}^{ \pm}\right)$(radius $R$ in formula (4)) between the center of the circumference generatrix and the rotation axis (point $x_{b}^{ \pm}$) changes from $G^{ \pm}$for $\chi=0$ to $H^{ \pm}$for $\chi=\chi_{n}^{ \pm}$.

The distance is $\left(0, x_{b}^{ \pm}\right)=G^{ \pm}$. The parameter $G^{ \pm}$is defined by the relations 


$$
G^{+}=\left\{\begin{array}{c}
D^{+} \text {if } \chi_{n}^{+} \geq \chi_{n}^{-} \\
D^{-} \frac{\tan \chi_{n}^{-}}{\tan \chi_{n}^{+}} \text {if } \chi_{n}^{+}<\chi_{n}^{-}
\end{array}, G^{-}=\left\{\begin{array}{c}
D^{+} \frac{\tan \chi_{n}^{+}}{\tan \chi_{n}^{-}} \text {if } \chi_{n}^{+} \geq \chi_{n}^{-} \\
D^{-} \text {if } \chi_{n}^{+}<\chi_{n}^{-}
\end{array},\right.\right.
$$

where $D^{ \pm}=\left[(1+\alpha) \bar{R}_{\text {out }}-R_{\text {in }}\right] \frac{\cos \chi_{n}^{ \pm}}{1-\cos \chi_{n}^{ \pm}}$. The output radius is $\bar{R}_{\text {out }}=\max \left(R_{\text {out }}^{-}, R_{\text {out }}^{+}\right)+\tau \sigma R_{\text {in }}$, where the parameter $0 \leq \tau \leq 1$ is chosen arbitrarily.

The upper torus $\left(0, x_{a}^{ \pm} f_{a}^{ \pm}, f_{c}^{ \pm}\right)$is described by the relations

$$
\begin{aligned}
& X^{ \pm U}(\varphi, \chi)= \pm\left[G^{ \pm}+X\left(\varphi, \chi-\pi, R^{M}\left(1, \chi ; 0, \chi_{n}^{ \pm}, G^{ \pm}, H^{ \pm}\right), R^{M}\left(1, \chi ; 0, \chi_{n}^{ \pm}, R_{\text {in }}, R_{\text {out }}^{ \pm}\right)\right)\right], \\
& Y^{ \pm U}(\varphi, \chi)=Y\left(\varphi, R^{M}\left(1, \chi ; 0, \chi_{n}^{ \pm}, R_{\text {in }}, R_{\text {out }}^{ \pm}\right)\right), \\
& Z^{ \pm U}(\varphi, \chi)=Z\left(\varphi, \chi-\pi, R^{M}\left(1, \chi ; 0, \chi_{n}^{ \pm}, G^{ \pm}, H^{ \pm}\right), R^{M}\left(1, \chi ; 0, \chi_{n}^{ \pm}, R_{\text {in }}, R_{\text {out }}^{ \pm}\right)\right),
\end{aligned}
$$

where $H^{ \pm}=G^{ \pm} / \cos \left(\chi_{n}^{ \pm}\right)-\alpha \bar{R}_{\text {out }}$ and $\varphi \in[\pi / 2,3 \pi / 2], \chi \in\left[0, \chi_{n}^{ \pm}\right]$.

The lower torus $\left(z_{a}, f_{c}^{ \pm}, f_{b}^{ \pm}, z_{b}\right)$ has the input radius of the generatrix $\left(f_{c}^{ \pm}, f_{b}^{ \pm}\right)=R_{\text {out }}^{ \pm}$and the output radius $\left(z_{a}, z_{b}\right)=\bar{R}_{\text {out }}$, the torus is bounded by the angle $\angle\left(z_{b} z_{d} f_{b}^{ \pm}\right)$. The distance along the arc $\left(z_{a}, f_{c}^{ \pm}\right)$between the center of the circumference generatrix and the rotation axis (point $z_{d}$ ) is $\alpha \bar{R}_{\text {out }}$. The right and left halves of the lower torus are matched on the line $\left(z_{a}, z_{d}\right)=\alpha \bar{R}_{\text {out }}$. The parameter $\alpha>\frac{1-\left[\max \left(R_{\text {out }}^{-}, R_{\text {out }}^{+}\right) / R_{\text {in }}+\tau\right]}{\left[\min \left(R_{\text {out }}^{-}, R_{\text {out }}^{+}\right) / R_{\text {in }}+\tau\right] \cdot \min \left(\cos \chi_{n}^{-}, \cos \chi_{n}^{+}\right)}$determines the radius of the lower rounding of the bronchus bifurcation. The parameters for a symmetric bronchial tree in [2] are $\alpha=1.5$ and $\tau=0$.

The lower torus $\left(z_{a}, f_{c}^{ \pm}, f_{b}^{ \pm}, z_{b}\right)$ is described by the relations

$$
\begin{aligned}
& X^{ \pm D}(\varphi, \chi)= \pm X\left(\varphi, B^{ \pm}(\chi), \alpha \bar{R}_{\text {out }}, R_{\text {out }}^{ \pm}\right), \\
& Y^{ \pm D}(\varphi, \chi)=Y\left(\varphi, R^{M}\left(1, B^{ \pm}(\chi) ; \chi_{n}^{ \pm}, \frac{\pi}{2}, R^{M}\left(1, \chi ; 0, \chi_{n}^{ \pm}, R_{\text {in }}, R_{\text {out }}^{ \pm}\right), \bar{R}_{\text {out }}\right)\right), \\
& Z^{ \pm D}(\varphi, \chi)=-G^{ \pm} \tan \chi_{n}^{ \pm}+Z\left(\varphi, B^{ \pm}(\chi), \alpha \bar{R}_{\text {out }}, R^{M}\left(1, B^{ \pm}(\chi) ; \chi_{n}^{ \pm}, \frac{\pi}{2}, R_{\text {out }}^{ \pm}, \bar{R}_{\text {out }}\right)\right),
\end{aligned}
$$

where $\varphi \in[\pi / 2,3 \pi / 2]$ and $\chi \in\left[\chi_{z}^{ \pm}, \chi_{n}^{ \pm}\right]$.

The function $B^{ \pm}(\chi)$ recalculates the angle with the point $z_{d}$ as center for design of lower torus to the angle $\chi$ with the point $x_{b}^{ \pm}$as center

$$
B^{ \pm}(\chi)=\chi+\arcsin \left(G^{ \pm} \cos (\chi) \frac{\tan \left(\chi_{n}^{ \pm}\right)-\tan (\chi)}{\alpha \bar{R}_{\text {out }}}\right) .
$$

The upper two-parameter surface $\left(0, z_{c}^{ \pm}, z_{a}\right)$ is defined by the equations 


$$
\begin{aligned}
& x^{ \pm U}(s)= \pm s, \\
& y^{ \pm U}(j, s, \chi)=R^{M}\left(\beta, s ; 0, \pm X^{ \pm U}\left(j \frac{\pi}{2}, \chi\right), j \cdot C^{ \pm}(\beta, \gamma, \chi), Y^{ \pm U}\left(j \frac{\pi}{2}, \chi\right)\right), \\
& z^{ \pm U}(s, \chi)=\left(s-G^{ \pm}\right) \tan \chi
\end{aligned}
$$

where $s \in\left[0, \pm X^{ \pm U}(j \cdot \pi / 2, \chi)\right]$ and $\chi \in\left[0, \chi_{z}^{ \pm}\right], j=-1,1$.

The function $C_{n}^{ \pm}(\beta, \gamma, \chi)=R^{M}\left(\beta, \chi^{\gamma} ; 0,\left(\chi_{z}^{ \pm}\right)^{\gamma}, R_{\text {in }}, \bar{R}_{\text {out }}\right)$ yields the equation for the line $\left(0, z_{a}\right)$ (see Fig. 1) in the plane $x=0$. The parameter $\gamma(\gamma \geq 1)$ controls the curvature of the line $\left(0, z_{a}\right)$ : for $\gamma=1$, the line is straight $(\beta=0)$ or sinusoidal $(\beta=1)$; for $\gamma>1$, it is a convex line $(\beta=0)$ or a convex sinusoid $(\beta=1)$.

The lower two-parameter surface $\left(0, z_{e}, z_{a}\right)$ is defined by the equations

$$
\begin{aligned}
x^{ \pm D}(s)= \pm s, & \\
y^{ \pm D}(s, \chi)= & R^{M}\left(\beta, s ; \pm X_{n}^{ \pm D}(j \cdot \pi / 2, \chi), \pm X^{ \pm U}(j \cdot \pi / 2, \chi),\right. \\
& \left.Y^{ \pm D}(j \cdot \pi / 2, \chi), Y^{ \pm U}(j \cdot \pi / 2, \chi)\right), \\
z^{ \pm D}(s, \chi)= & \left(s-G^{ \pm}\right) \tan (\chi),
\end{aligned}
$$

where $s \in\left[ \pm X^{ \pm D}(j \cdot \pi / 2, \chi), \pm X^{ \pm U}(j \cdot \pi / 2, \chi)\right]$ and $\chi \in\left[\chi_{z}^{ \pm}, \chi_{n}^{ \pm}\right], j=-1,1$.

The parameter $\beta$ in the formulas for the two-parameter surfaces (10) and (11) controls the smoothness of matching of these surfaces with the upper and lower torus configurations (7) and (8). For $\beta=1$, matching has the second order of smoothness (without discontinuities of derivatives); for $\beta=0$, surfaces (10) and (11) are constructed as flat as possible, but the angles of their matching with the torus configurations (5) is not equal to zero. As a result, the bifurcation design in acute curvilinear angles $\angle\left(f_{b}^{ \pm} z_{a} f_{c}^{ \pm}\right)$and $\angle\left(z_{a} 0 z_{c}^{ \pm}\right)$is simplified to the greatest possible extent (see Fig. 1).

The general view of the resultant three-dimensional bifurcation with circular and starry cross sections of the bronchi is shown in Figure 2.

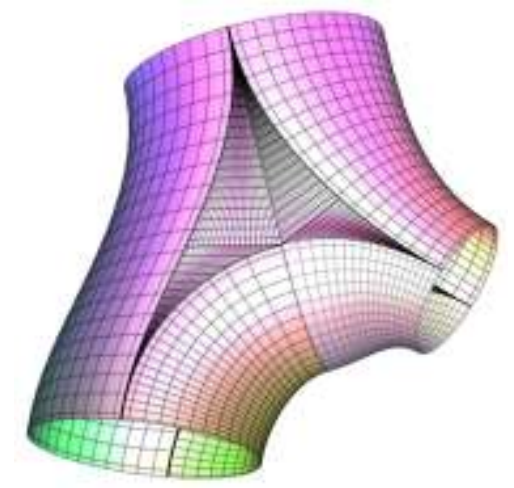

a)

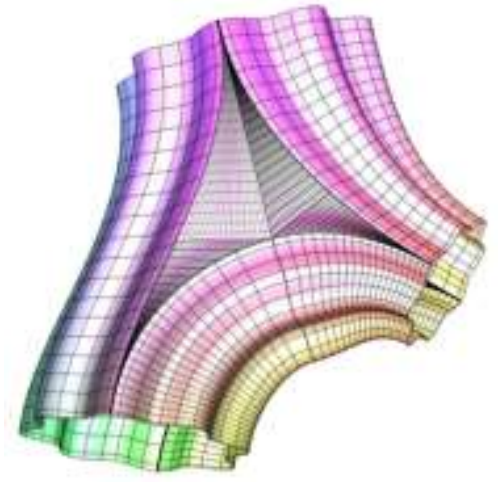

b)

Fig. 2. General view of the three-dimensional asymmetric bifurcation with circular (a) and starry (b) cross sections of the bronchi. 
The output bronchi are adjacent to the bifurcation at the points $f_{c}^{ \pm}$, which are the centers of the output bronchi. The coordinates of the points $f_{c}^{ \pm}$are defined as

$$
\begin{aligned}
& x^{ \pm c}= \pm \alpha \bar{R}_{\text {out }} \cos \chi_{n}^{ \pm}, \\
& y^{ \pm c}=0, \\
& z^{ \pm c}=-H^{ \pm} \sin \chi_{n}^{ \pm} .
\end{aligned}
$$

The surface $\left(f_{a}^{ \pm}, f_{c}^{ \pm}, f_{b}^{ \pm}\right)$is adjacent to the output cylindrical or starry bronchus of length $L_{\text {out }}^{ \pm}$(not shown in Fig. 1). The equations of the output bronchi are

$$
\begin{aligned}
& X^{ \pm B(\text { out })}(\varphi, s)=x^{ \pm c}+R_{\text {out }}^{ \pm} f(\varphi) \cos (\varphi) \cos \chi_{n}^{ \pm} \pm s \cdot \sin \chi_{n}^{ \pm}, \\
& Y^{ \pm B(\text { out })}(\varphi, s)=R_{\text {out }}^{ \pm} f(\varphi) \sin (\varphi), \\
& Z^{ \pm B \text { (out) }}(\varphi, s)=z^{ \pm c} \pm R_{\text {out }}^{ \pm} f(\varphi) \cos (\varphi) \sin \chi_{n}^{ \pm}-s \cdot \cos \chi_{n}^{ \pm},
\end{aligned}
$$

where the plus and minus signs refer to the right and left output bronchi, respectively, the parameter is $s \in\left[0, L_{\text {out }}^{ \pm}\right]$, and the angle is $\varphi \in[0,2 \pi]$.

Equations (4)-(13) provide an analytical description of the construction of the bronchial tree bifurcation with the output bronchi. Each bifurcation consists of the following 3D surfaces: (1) two upper toroidal surfaces (formulas (7)); (2) two lower toroidal surfaces (formulas (8)); (3) four upper two-parameter surfaces (formulas (10)); four lower two-parameter surfaces (formulas (11)); two output cylindrical or starry bronchi (formulas (13)). Here the plus and minus signs refer to the right half $(x>0)$ and left half $(x<0)$, respectively. The subscripts are $j=1$ for the coordinates $y>0$ and $j=-1$ for the coordinate $y<0$.

\section{Construction of bronchus bending}

The first stage of a close-to-real bronchial tree construction, is the design of the region of an individual bronchus bending.

This process uses modified formulas (7) to design the upper torus

$$
\begin{aligned}
& X^{ \pm L}(\varphi, \chi)= \pm\left[G^{ \pm}+X\left(\varphi, \chi-\pi, R^{M}\left(1, \chi ; 0, \chi_{n}^{ \pm}, G^{ \pm}, H^{ \pm}\right), R^{M}\left(1, \chi ; 0, \chi_{n}^{ \pm}, R_{\text {in }}, R_{\text {out }}^{ \pm}\right)\right)\right], \\
& Y^{ \pm L}(\varphi, \chi)=Y\left(\varphi, R^{M}\left(1, \chi ; 0, \chi_{n}^{ \pm}, R_{n}, R_{n+1}^{ \pm}\right)\right), \\
& Z^{ \pm L}(\varphi, \chi)=Z\left(\varphi, \chi-\pi, R^{M}\left(1, \chi ; 0, \chi_{n}^{ \pm}, G^{ \pm}, H^{ \pm}\right), R^{M}\left(1, \chi ; 0, \chi_{n}^{ \pm}, R_{\text {in }}, R_{\text {out }}^{ \pm}\right)\right),
\end{aligned}
$$

where $\varphi \in[\pi / 2,5 \pi / 2]$ and $\chi \in\left[0, \chi_{n}^{ \pm}\right]$.

Only one of the branches (right or left branch indicated by the plus or minus sign, respectively) becomes bent; correspondingly, only one sign (plus or minus) is used in formulas (14). Bronchus bending is considered as a half-bifurcation with one branch. To have formulas of the same form, it is formally assumed that $R_{\text {out }}^{+}=R_{\text {out }}^{-}, \chi_{n}^{+}=\chi_{n}^{-}$, etc.

\section{Construction of the bronchial tree}

Bronchial tree construction starts from the upper bifurcation, followed by consecutive branching of the bronchial tree. To construct the bronchial tree as a sequence of bifurcations, one shall transform the coordinate system, and design bifurcations in the new coordinate system by formulas (6)-(14). Construction of the following bifurcations of the bronchial tree starts from the transformation of the coordinate system with the origin at the center of the right or left output bronchus of the previous bifurcation. The $Z$ axis is directed along the axis of the left 
(for the left sub-branch of the tree) or right (for the right sub-branch) output bronchus of the previous bifurcation. This transformation of coordinates allows a researcher to transfer the bifurcation to an appropriate place in the tree without changing the formulas for bronchus design.

Let us progress to the new coordinate system from the current bifurcation when the origin of the coordinate axes is shifted to the point $\left(x^{ \pm \text {new }}, y^{ \pm \text {new }}, z^{ \pm \text {new }}\right)$ :

$$
\begin{aligned}
& x^{ \pm \text {new }}= \pm\left(\alpha R_{\text {out }} \cos \chi_{n}^{ \pm}+L_{\text {out }} \sin \chi_{n}^{ \pm}\right), \\
& y^{ \pm \text {new }}=0, \\
& z^{ \pm \text {new }}=-H^{ \pm} \sin \chi_{n}^{ \pm}-L_{\text {out }} \cos \chi_{n}^{ \pm} .
\end{aligned}
$$

Then the coordinate system is rotated by the angle $\chi_{n}^{ \pm}$around the $Y$ axis and by the angle $\sigma_{n}$ around the $Z$ axis. All rotations are performed in the clockwise direction.

In starry bronchus design, the angle of rotation around the $Z$ axis is subjected to the condition $\sigma_{n}=2 k \pi / n_{\text {ed }}$ (see the formulas for the star (1) and (2)), where $k \geq 0$ is an arbitrary integer number. When this requirement is fulfilled, the rays of the input bifurcation star after its rotation coincide with the rays of the star of the output bronchus of the previous bifurcation. For example, if the rotation angle is $\sigma_{n}=\pi / 2$, the number of rays should be multiple of four ( $\left.n_{\text {ed }}=4 k\right)$ as in [2].

The matrix of the coordinate transformation for the next bifurcation has the form

$$
S^{ \pm \text {new }}=\left[\begin{array}{cccc}
\cos \left(\sigma_{n}\right) \cdot \cos \left(\chi_{n}^{ \pm}\right) & -\sin \left(\sigma_{n}\right) & \pm \cos \left(\sigma_{n}\right) \cdot \sin \left(\chi_{n}^{ \pm}\right) & 0 \\
\sin \left(\sigma_{n}\right) \cdot \cos \left(\chi_{n}^{ \pm}\right) & \cos \left(\sigma_{n}\right) & \pm \sin \left(\sigma_{n}\right) \cdot \sin \left(\chi_{n}^{ \pm}\right) & 0 \\
\mp \sin \left(\chi_{n}^{ \pm}\right) & 0 & \cos \left(\chi_{n}^{ \pm}\right) & 0 \\
x^{ \pm \text {new }} & y^{ \pm \text {new }} & z^{ \pm \text {new }} & 1
\end{array}\right] .
$$

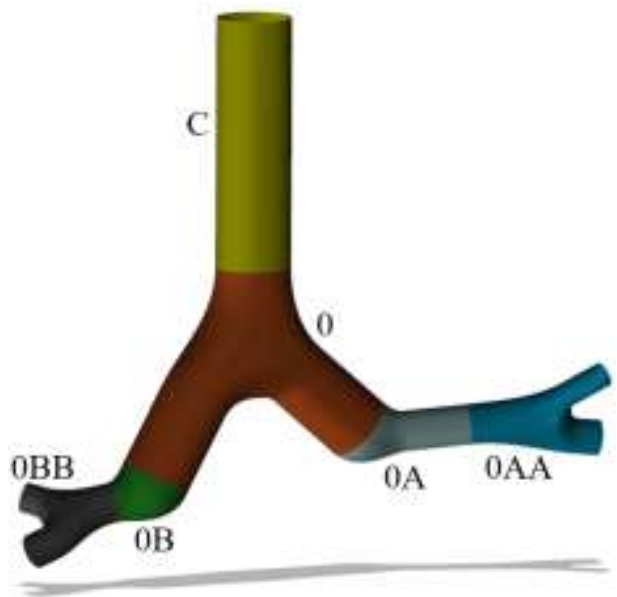

a)

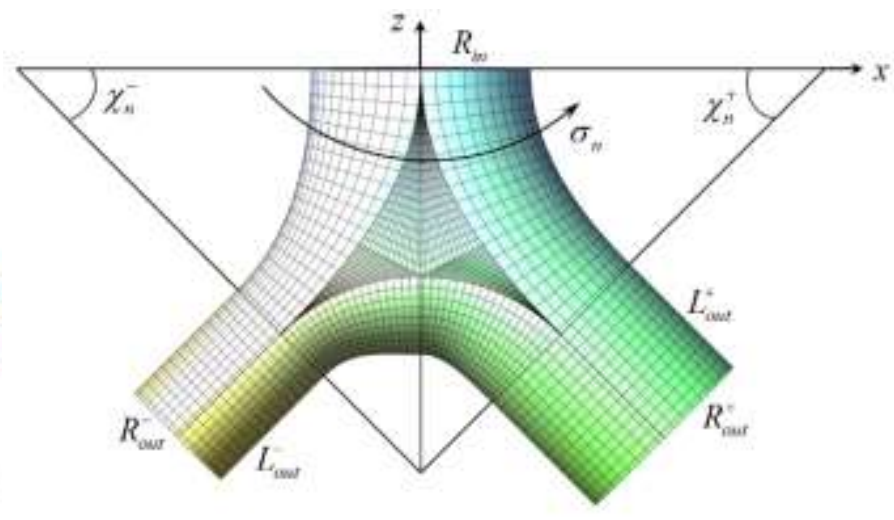

b)

Fig. 3. Example of design of an asymmetric bronchial tree (a). Setting the initial parameters of the bifurcation (b).

Figure $3 \mathrm{a}$ is an example of an asymmetric bronchial tree construction. Here different bifurcations are indicated by different colors. The scheme of the input parameters of the bifurcation is shown in Figure 3b. The parameters of bifurcations from Figure $3 \mathrm{a}$ are listed in Table 1. The designed bronchial tree consists of the input bronchus $C$ of $100 \mathrm{~mm}$ length and 
$15 \mathrm{~mm}$ radius. It is convenient to denote the bifurcations by $0 \mathrm{~A}, 0 \mathrm{~B}$, etc., where A refers to the right branch $(i=1)$ and $\mathrm{B}$ refers to the left branch $(i=-1)$.

Bronchus bending (with no bifurcation) is constructed by formulas (14) - half-bifurcation (these are bifurcations $0 \mathrm{~A}$ and $\mathrm{OB}$ in Fig. 3a). When the next bronchus is constructed by the coordinate transformation formulas (16), the $Z$ axis of the new coordinate system passes along the axis of the output bronchus. To turn and smoothly match the next bifurcation off the output bronchus axis, a half-bifurcation with a zero length of the output bronchus is used (this is bifurcation $0 \mathrm{~B}$ in Fig. 3a and Table 1). Such a method allows a researcher to construct a bronchial tree of arbitrary complexity with the use of unified formulas.

Table 1. Parameters of the bronchial tree*

\begin{tabular}{|c|c|c|c|c|c|c|c|c|c|c|}
\hline Bifurcation & $i$ & $\begin{array}{c}R_{\text {in }}, \\
\mathrm{mm}\end{array}$ & $\begin{array}{c}L_{\text {out }}^{+}, \\
\mathrm{mm}\end{array}$ & $\begin{array}{c}L_{\text {out }}^{-}, \\
\mathrm{mm}\end{array}$ & $\begin{array}{c}R_{\text {out }}^{+}, \\
\mathrm{mm}\end{array}$ & $\begin{array}{c}R_{\text {out }}^{-}, \\
\mathrm{mm}\end{array}$ & $\begin{array}{c}\chi_{n}^{+}, \\
\mathrm{deg} .\end{array}$ & $\begin{array}{c}\chi_{n}^{-}, \\
\mathrm{deg} .\end{array}$ & $\begin{array}{c}\sigma_{n}, \\
\mathrm{deg} .\end{array}$ & $\begin{array}{c}\text { Bifurcation } \\
\text { type }\end{array}$ \\
\hline 0 & $+1,-1$ & 15 & 35 & 35 & 14 & 14 & 50 & 30 & 0 & Full \\
\hline 0A & -+1 & 14 & 25 & - & 8 & - & 50 & - & 30 & Half \\
\hline 0B & -1 & 14 & - & 0 & - & 8 & - & 50 & 10 & Half \\
\hline 0AA & $+1,-1$ & 8 & 17 & 7 & 5 & 7 & 35 & 15 & 20 & Full \\
\hline 0BB & $+1,-1$ & 8 & 7 & 6 & 7 & 6 & 15 & 45 & 55 & Full \\
\hline
\end{tabular}

${ }^{*} \alpha=2, \beta=0, \gamma=2, \tau=0.1$.

\section{CONCLUSIONS}

The article presents a method designing the geometry of a morphologically realistic human bronchial tree. The method is important because it provides a possibility to set parametrically the bronchus constriction at a level maximally close to the real condition at the obstructive pulmonary diseases.

The design of the bronchial tree bifurcation for different bronchus sizes and different angles of bronchi branching is performed with the use of the developed analytical formulas. The degree of bronchi pathology is defined by an analytical dependence, which describes close-to-real starry constriction of the bronchi. Replacement of formula (1), which describes bronchus constriction, by another analytical formula allows a researcher to model some other (not starry) types of constriction of pathological bronchi.

The proposed method of bronchial tree design is based on a unified mathematical model of bifurcation construction. Bronchus bending is described as a particular case of the bifurcation (half-bifurcation), and the bronchi can be turned in space by an arbitrary angle. The transition to the next bifurcation is performed by means of a simple transformation of the local coordinate system.

One of the key features of the method is its usability without monotonic manual operations for constructing each individual bifurcation and matching of these bifurcations in 3D simulation codes. This property ensures repeatability of results, i.e., the bronchial tree designed by one researcher can be easily repeated by other researchers. This repeatability was not possible before because of the large labor effort necessary for a bronchial tree design and because of the differences associated with using particular codes of 3D simulations. Different 3D codes have their own rules of matching curves and surfaces, which finally affects the resultant bronchial tree design. When the of bifurcation is large, the differences are summarized, resulting in error accumulation at an attempt to repeat the bronchial tree design with another 3D code. To the author's knowledge, the bronchial tree with the starry pathology of the bronchi is not designed up to now because it is extremely complicated. 
The proposed analytical formulas for bronchial tree design allow one to perform numerical simulations of bronchial trees of an arbitrary generation (certainly, if appropriate computational resources are available) for healthy and pathological bronchi.

The study was supported by the Russian Foundation for Basic Research and by the Subject of the Russian Federation (Novosibirsk Region) within the framework of the research project No. 19-41540003 r_a.

\section{REFERENCES}

1. Weibel E.R. Morphometry of the Human Lung. Springer Verlag, Berlin, 1963.

2. Medvedev A.E., Gafurova P.S. Analytical design of the human bronchial tree for healthy patients and patients with obstructive pulmonary diseases. Mathematical Biology and Bioinformatics. 2019. V. 14. No. 2. P. 635-648. doi: 10.17537/2019.14.635.

3. Zhao Y., Lieber B.B. Steady inspiratory flow in a model symmetric bifurcation. ASME Journal of Biomechanical Engineering. 1994. V. 116. P. 488-496.

4. Zhao Y., Brunskill C.T., Lieber B.B. Inspiratory and expiratory steady flow analysis in a model symmetrically bifurcating airway. ASME Journal of Biomechanical Engineering. 1997. V. 119. P. 52-58.

5. Hegedüs C.J., Balásházy I., Farkas Á. Detailed mathematical description of the geometry of airway bifurcations. Respiratory physiology \& neurobiology. 2004. V. 141. No. 1. P. 99-114. doi: 10.1016/j.resp.2004.03.004.

6. Heistracher T., Hofmann W. Physiologically realistic models of bronchial airway bifurcations. J. Aerosol Sci. 1995. V. 26. No. 3. P. 497-509.

7. Ertbruggen C., Hirsch C., Paiva M. Anatomically based three-dimensional model of airways to simulate flow and particle transport using computational fluid dynamics. J. Appl. Physiol. 2005. V. 98. P. 970-980. doi: 10.1152/japplphysiol.00795.2004.

8. Tena A.F., Casan P., Fernández J., Ferrera C., A. Marcos A. Characterization of particle deposition in a lung model using an individual path. EPJ Web of Conferences. 2013. V. 45. Article No. 01079. doi: 10.1051/epjconf/20134501079.

9. Tena A.F., Fernández J., Álvarez E., Casan P., Walters D.K. Design of a numerical model of lung by means of a special boundary condition in the truncated branches. International Journal for Numerical Methods in Biomedical Engineering. 2017. V. 33. No. 6. Article No. e2830. doi: 10.1002/cnm.2830.

10. Tena A.F., Francos J.F., Álvarez E., Casan P.A. A three dimensional in SILICO model for the simulation of inspiratory and expiratory airflow in humans. Engineering Applications of Computational Fluid Mechanics. 2015. V. 9. No. 1. P. 187-198. doi: 10.1080/19942060.2015.1004819.

11. Gemci T., Ponyavin V., Chen Y., Chen H., Collins R. CFD Simulation of Airflow in a 17-Generation Digital Reference Model of the Human Bronchial Tree. Series on Biomechanics. 2007. V. 23. No. 1. P. 5-18.

12. Gemci T., Ponyavin V., Chen Y., Chen H., Collins R. Computational model of airflow in upper 17 generations of human respiratory tract. Journal of Biomechanics. 2008. V. 41. P. 2047-2054. doi: 10.1016/j.jbiomech.2007.12.019.

13. Trusov P.V., Zaitseva N.V., Tsinker M.Yu. Modeling of human breath: conceptual and mathematical statements. Mathematical Biology and Bioinformatics. 2016. V. 11. No 1. P. 64-80. doi: 10.17537/2016.11.64.

14. Trusov P.V., Zaitseva N.V., Tsinker M.Yu., Babushkina A.V. Modelling dusty air flow in the human respiratory tract. Ross. Zh. Biomekhaniki. 2018. V. 22. No. 3. P. 301-314. doi: 10.15593/RZhBiomeh/2018.3.03.

15. Choi J. Multiscale numerical analysis of airflow in CT-based subject specific breathing human lungs: PhD Dissertation. Iowa: University of Iowa, 2011. 259 p.

16. Ham A.W., Cormack D.H. Ham's Histology. Philadelphia, Lippencott, 1979. 
17. Anthony L. Mescher Junqueira's. Basic Histology: Text and Atlas. $13^{\text {th }}$ Edition. New York: McGraw Hill Medical, 2013. 560 p.

\title{
Методика построения несимметричного бронхиального дерева человека в норме и при патологии
}

\author{
Медведев А.Е.
}

Институт теоретической и прикладной механики им. С.А. Христиановича СО РАН, Новосибирск, Россия Новосибирский государственный университет, Новосибирск, Россия

\begin{abstract}
Аннотация. Работа посвящена аналитическому построению полного несимметричного бронхиального дерева (неправильная дихотомия) человека в норме и при обструктивной болезни легких. Приведены аналитические формулы для построения полного бронхиального дерева. Все поверхности бронхиального дерева состыковываются со вторым порядком гладкости (не имеют острых углов и ребер). Геометрические характеристики бронхиального дерева человека при патологии моделируются “звездчатой” формой внутреннего строения бронха, степень патологии задается параметрами степенью сужения бронхов и степенью искажения цилиндрической формы бронхов. Конечные аналитические формулы позволяют построит бронхиальное дерево человека любой сложности (вплоть до альвеол) при этом параметрические зависимости позволяют задать любую степень обструкции легких.
\end{abstract}

Ключевые слова: бронхиальное дерево, математическое моделирование, легкие человека, дыхательная система, бифуркаиия, болезнь легких. 